\title{
Bounds on the Probability of Success of Postselected Non-linear Sign Shifts Implemented with Linear Optics
}

\author{
E. Knill* \\ Los Alamos National Laboratory, MS B256, Los Alamos, NM 87545
}

(Dated: February 5, 2020)

\begin{abstract}
The fundamental gates of linear optics quantum computation are realized by using single photons sources, linear optics and photon counters. Success of these gates is conditioned on the pattern of photons detected without using feedback. Here it is shown that the maximum probability of success of these gates is typically strictly less than 1 . For the one-mode nonlinear sign shift, the probability of success is bounded by $1 / 2$. For the conditional sign shift of two modes, this probability is bounded by $3 / 4$. These bounds are still substantially larger than the highest probabilities shown to be achievable so far, which are $1 / 4$ and $2 / 27$, respectively.
\end{abstract}

\section{INTRODUCTION}

It has recently been shown that it is possible, in principle, to scalably quantum compute with single photon sources, linear optics and photon counters [1] ${ }^{1}$. Key elements in the scheme that makes this possible are optical gates that use helper photons, linear optics and postselection on specific photon counts to realize simple non-linear operations on one or more modes. Two such gates are the one-mode non-linear sign shift

$$
\mathrm{NS}: \alpha|0\rangle+\beta|1\rangle+\gamma|2\rangle \rightarrow \alpha|0\rangle+\beta|1\rangle-\gamma|2\rangle
$$

and the two-mode conditional sign shift

$$
\mathrm{CS}: \alpha|00\rangle+\beta|10\rangle+\gamma|01\rangle+\delta|11\rangle \rightarrow \alpha|00\rangle+\beta \| 10\rangle+\gamma|01\rangle-\delta|11\rangle .
$$

Here $|j\rangle$ is the state with $j$ photons in one mode and $|j k\rangle$ is the state with $j$ photons in the first and $k$ photons in the second mode. How these gates act on states other than those explicitly given does not matter for current purposes. To efficiently use these gates, one would like to implement them with as high a probability of success as possible. To do so one may use single helper photons in helper modes, apply a linear optics transformation (that is, a series of beam splitters and phase shifters), and a combination of photon counting measurements of the helper modes. In the remainder of this report, a procedure using single helper photons and linear optics is called an LOP procedure. LOP states are those obtained by an LOP procedure from the vacuum. Postselection based on measured photon counts is abbreviated as PC. All procedures considered here are assumed not to involve feedback from PC, that is, they consist of LOP followed by PC. Currently, the highest probabilities of success achieved for implementing NS and CS with LOP followed by PC are 1/4 [1] and 2/27 [4], respectively. What are the maximum probabilities of

\footnotetext{
*knill@lanl.gov

${ }^{1}$ See also [2] for an alternative approach and [3] for a significant improvement
} 
success $P_{\max }(\mathrm{NS})$ and $P_{\max }(\mathrm{CS})$ for realizing these gates with LOP followed by PC? In [4] it was shown that these probabilities cannot be exactly one. The main result of this report is to show that $P_{\max }(\mathrm{NS}) \leq 1 / 2$ and $P_{\max }(\mathrm{CS}) \leq 3 / 4$. To prove these bounds, the gates are used to create special two-photon states. The next step is to obtain upper bounds on the maximum overlaps of these states with states that can be generated with LOP. Since high probability of success for the gates implies high overlap with the state obtained just before postselection, the desired bounds can be obtained. The bounds on the overlaps are derived by considering photon statistics of LOP states. The techniques can be applied to obtain bounds on the probability of success of other postselected gates.

\section{UPPER BOUNDS: NS}

To bound $P_{\max }$ (NS) from above, assume that we can implement NS using LOP followed by PC with probability of success $p$. The following procedure creates the two photon state from single photon states with probability of success $p$ :

1. Prepare the state $|11\rangle_{a b}=\mathbf{a}^{\dagger(a)} \mathbf{a}^{\dagger(b)}|0\rangle$ consisting of one photon in each of modes $a$ and $b$. Here $|0\rangle$ is the vacuum state and $\mathbf{a}^{\dagger(x)}$ is the creation operator for mode $x$.

2. Set $\alpha=\cos (\pi / 8)$ and $\beta=\sin (\pi / 8)$. Use the beam splitter that transforms $|10\rangle_{\mathrm{ab}} \rightarrow$ $\alpha|10\rangle_{\mathrm{ab}}+\beta|01\rangle_{\mathrm{ab}}$ and $|01\rangle_{\mathrm{ab}} \rightarrow-\beta|10\rangle_{\mathrm{ab}}+\alpha|01\rangle_{\mathrm{ab}}$. Writing $U$ for the unitary operator implemented by this beam splitter, $U$ 's action can be derived from how it transforms the annihilation and creation operators for the modes. That is, $U \mathbf{a}^{\dagger\left(a^{(a)}\right.} U^{\dagger}=\alpha \mathbf{a}^{\dagger(a)}+\beta \mathbf{a}^{{ }^{(b)}}$ and $U \mathbf{a}^{\dagger(\mathrm{b})} U^{\dagger}=-\beta \mathbf{a}^{\left({ }^{(a)}\right.}+\alpha \mathbf{a}^{(\mathrm{b})}$, where $\mathbf{a}^{(\mathrm{l})}$ and $\mathbf{a}^{{ }^{(l)}}$ are the annihilation and creation operators for mode I, respectively. The following state is obtained after applying this beam splitter:

$$
\begin{aligned}
U|11\rangle_{\mathrm{ab}} & =U \mathbf{a}^{\dagger(\mathrm{a})} \mathbf{a}^{\dagger(\mathrm{b})}|\mathbf{0}\rangle \\
& =U \mathbf{a}^{\dagger(\mathrm{a})} U^{\dagger} U \mathbf{a}^{\dagger(\mathrm{b})} U^{\dagger} U|\mathbf{0}\rangle \\
& \left.=\left(\alpha \mathbf{a}^{\dagger(\mathrm{a})}+\beta \mathbf{a}^{\dagger(\mathrm{b})}\right)\left(-\beta \mathbf{a}^{\dagger(\mathrm{a})}+\alpha \mathbf{a}^{\dagger(\mathrm{b})}\right) \| \mathbf{0}\right\rangle \\
& =\left(-\alpha \beta \mathbf{a}^{\dagger(\mathrm{a})^{2}}+\left(\alpha^{2}-\beta^{2}\right) \mathbf{a}^{\dagger(\mathrm{a})} \mathbf{a}^{\dagger(\mathrm{b})}+\alpha \beta \mathbf{a}^{\dagger(\mathrm{b})^{2}}\right)|0\rangle \\
& =-\sqrt{2} \alpha \beta|20\rangle_{\mathrm{ab}}+\left(\alpha^{2}-\beta^{2}\right)|11\rangle_{\mathrm{ab}}+\sqrt{2} \alpha \beta|02\rangle .
\end{aligned}
$$

3. Apply NS to mode a to obtain

$$
\begin{aligned}
\sqrt{2} \alpha \beta|20\rangle_{\mathrm{ab}}+( & \left.\left.\alpha^{2}-\beta^{2}\right) \| 11\right\rangle_{\mathrm{ab}}+\sqrt{2} \alpha \beta|02\rangle_{\mathrm{ab}} \\
& =\frac{1}{2}\left(\sin (\pi / 4) \mathbf{a}^{\dagger(\mathrm{a})^{2}}+2 \cos (\pi / 4) \mathbf{a}^{\dagger(\mathrm{a})} \mathbf{a}^{\dagger(\mathrm{b})}+\sin (\pi / 4) \mathbf{a}^{\dagger(\mathrm{b})^{2}}\right)|\mathbf{0}\rangle \\
& =\frac{1}{\sqrt{2}}\left(\frac{1}{\sqrt{2}}\left(\mathbf{a}^{\dagger(\mathrm{a})}+\mathbf{a}^{\dagger(\mathrm{b})}\right)\right)^{2}|\mathbf{0}\rangle
\end{aligned}
$$

with probability of success $p$. Here, $|0\rangle$ is the vacuum state, that is, the state with no photons in any of the modes under consideration.

4. By using a $50 / 50$ beam splitter that maps $\left.\frac{1}{\sqrt{2}}(\| 10\rangle_{\mathrm{ab}}+|01\rangle_{\mathrm{ab}}\right) \rightarrow|10\rangle_{\mathrm{ab}}$, the state $\left.\frac{1}{\sqrt{2}} \mathbf{a}^{\dagger(a)^{2}} \| \mathbf{0}\right\rangle=|20\rangle_{\mathrm{ab}}$ is obtained. 
The effect of the above procedure is unchanged if PC is delayed until the end. Let $\rho$ be the final state (density matrix) on mode a just before postconditioning. Because postconditioning on a measurement of modes other than a to obtain $|2\rangle_{a}^{a}(2 \mid$ is possible and the probability of success is $p, \rho$ can be expressed as a mixture $p|2\rangle_{+}^{+}\langle 2|(1-p) \rho^{\prime}$ for some state $\rho^{\prime}$. To bound $p$ from above requires the following result:

Theorem 1 Let $\varrho$ be an LOP state. Then @'s expected number of photons in any mode is at most 1 .

The expected number of photons in mode a for $\rho$ is given by $2 p+x$, where $x \geq 0$. It follows that $p \leq 1 / 2$, establishing the desired bound on $P_{\max }$ (NS).

Proof of Theorem $\square$ Let the initial state before applying the linear optics transformation be given by

$$
|\psi\rangle=\mathbf{a}^{\dagger(1)} \ldots \mathbf{a}^{\dagger(\mathrm{k})}|0\rangle,
$$

where $k$ is the number of single photons used. Let the linear optics transformation $U$ act on modes 1 through $\mathrm{n}, \mathrm{n} \geq \mathrm{k}$. The transformation is completely determined by its $n \times n$ unitary matrix $\hat{U}=\left(u_{\mathrm{j}}\right)$ determined by $U^{\dagger} \mathbf{a}^{\dagger(1)} U=\sum_{\mathrm{j}} u_{\mathrm{j}} \mathbf{a}^{\mathbf{a}^{(\mathrm{j})}}[5]$. Without loss of generality, consider the expected number of photons in the first mode after $U$ has been applied. Compute

$$
\begin{aligned}
\left\langle\mathbf{n}^{(1)}\right\rangle & =\left\langle\psi\left|U^{\dagger} \mathbf{a}^{\dagger(1)} \mathbf{a}^{(1)} U\right| \psi\right\rangle \\
& =\left\langle\psi\left|U^{\dagger} \mathbf{a}^{\dagger(1)} U U^{\dagger} \mathbf{a}^{(1)} U\right| \psi\right\rangle \\
& =\left\langle\psi\left|\left(\sum_{j} u_{j 1} \mathbf{a}^{(j)}\right)\left(\sum_{l} \bar{u}_{11} \mathbf{a}^{(1)}\right)\right| \psi\right\rangle \\
& =\sum_{j \mid} u_{j 1} u_{l 1}\left\langle\psi\left|\mathbf{a}^{\dagger(j)} \mathbf{a}^{(1)}\right| \psi\right\rangle \\
& =\sum_{j=1}^{k}\left|u_{j 1}\right|^{2} \\
& \leq 1 .
\end{aligned}
$$

The second last step follows because $|\psi\rangle$ has well defined photon numbers in each mode, with none in modes beyond mode $\mathrm{k}$. The last step follows by unitarity of $\hat{U}$.

\section{UPPER BOUNDS: CS}

The bound on $P_{\max }(\mathrm{CS})$ is obtained in the same way as that on $P_{\max }(\mathrm{NS})$. Assume that we can implement CS with probability of success $p$. The first step is to show that one can create a state with expected number of photons $4 / 3$ in a mode using one instance of $\mathrm{C}$.

1. Prepare the state $|110\rangle_{\mathrm{abc}}$.

2. Use a beam splitter on modes $b$ and $c$ to make the state

$$
\frac{1}{\sqrt{3}}|110\rangle_{\mathrm{abc}}+\frac{\sqrt{2}}{\sqrt{3}}|101\rangle_{\mathrm{abc}} .
$$


3. Use a beam splitter on modes a and b that transforms $U_{1}|10\rangle=\cos (\pi / 8)|10\rangle_{\mathrm{ab}}-$ $\sin (\pi / 8)|01\rangle_{\mathrm{ab}}$ and $U_{1}|01\rangle=\sin (\pi / 8)|10\rangle_{\mathrm{ab}}+\cos (\pi / 8)|01\rangle_{\mathrm{ab}}$. This gives

$$
\frac{1}{\sqrt{3}} U_{1}|110\rangle_{\mathrm{abc}}+\frac{\sqrt{2}}{\sqrt{3}}\left(\cos (\pi / 8)|101\rangle_{\mathrm{abc}}-\sin (\pi / 8)|011\rangle_{\mathrm{abc}}\right) .
$$

4. Apply CS on to modes b and $\mathrm{c}$ with probability of success $p$ to obtain

$$
\frac{1}{\sqrt{3}} U_{1}|110\rangle_{\mathrm{abc}}+\frac{\sqrt{2}}{\sqrt{3}}\left(\cos (\pi / 8)|101\rangle_{\mathrm{abc}}+\sin (\pi / 8)|011\rangle_{\mathrm{abc}}\right) .
$$

5. Apply the inverse of the beam splitter used in step 3. The state is now

$$
\begin{aligned}
&|\psi\rangle= \frac{1}{\sqrt{3}}|110\rangle_{\mathrm{abc}}+\frac{\sqrt{2}}{\sqrt{3}}\left(\left(\cos (\pi / 8)^{2}-\sin (\pi / 8)^{2}\right)|101\rangle_{\mathrm{abc}}\right. \\
&\left.+2 \cos (\pi / 8) \sin (\pi / 8)|011\rangle_{\mathrm{abc}}\right) \\
&=\frac{1}{\sqrt{3}}\left(|110\rangle_{\mathrm{abc}}+|101\rangle_{\mathrm{abc}}+|011\rangle_{\mathrm{abc}}\right) .
\end{aligned}
$$

The claim is that the logical mode associated with annihilation operator $\mathbf{a}^{(1)}=\frac{1}{\sqrt{3}}\left(\mathbf{a}^{(\mathrm{a})}+\mathbf{a}^{(\mathrm{b})}+\mathbf{a}^{(\mathrm{c})}\right)$ has expected photon number $4 / 3$. This logical mode can be transformed into mode a by a linear optics transformation. Using Thm. 1 we can conclude, as before, that the maximum probability with which this state can be obtained is $3 / 4$. To prove the claim compute

$$
\begin{aligned}
\left\langle\psi\left|\mathbf{n}^{(1)}\right| \psi\right\rangle & =\left\langle\psi\left|\mathbf{a}^{\dagger^{(1)}} \mathbf{a}^{(1)}\right| \psi\right\rangle \\
= & \frac{1}{3}\langle\psi|\left(\mathbf{a}^{(a)}+\mathbf{a}^{(\mathrm{b})}+\mathbf{a}^{(\mathrm{c})}\right)\left(\mathbf{a}^{\dagger(\mathrm{a})}+\mathbf{a}^{\dagger(\mathrm{b})}+\mathbf{a}^{\dagger}(\mathrm{c})|\psi\rangle\right. \\
= & \frac{1}{3}\left(\frac{2}{\sqrt{3}}\left({ }^{\mathrm{abc}}\langle 100|+{ }^{\mathrm{abc}}\langle 010|+{ }^{\mathrm{abc}}\langle 001|\right)\right. \\
& \left.\quad \frac{2}{\sqrt{3}}\left(|100\rangle_{\mathrm{abc}}+|010\rangle_{\mathrm{abc}}+|001\rangle_{\mathrm{abc}}\right)\right) \\
= & \frac{4}{3} .
\end{aligned}
$$

\section{DISCUSSION}

The above results reduce the bounds on the probabilities of success of NS and CS using LOP followed by PC to values strictly below 1 . However, the gap between the highest probability of the known procedures and the bounds found is still large. An obvious reason that the bounds found here are probably not optimal is that they are insensitive to the type of measurement device used to implement the postselection. That is, it doesn't matter whether a photon counter or any arbitrarily more powerful measurement device is used, the bounds are still valid. Nevertheless, better bounds on the probabilities of success may be obtainable without using properties of photon counters. For example, it may be possible to obtain better bounds by using the NS and CS gates one or more times to obtain states that are further from LOP states. Note that the two photon state can be 
obtained with probability $1 / 2$ with LOP followed by PC: Apply a 50/50 beam splitter to the state $|11\rangle$ and condition on measuring no photons in the second mode. Here is an example of states that can be investigated: It is not hard to see that with one application of CS to a state obtained from $|11\rangle$, one can make the entangled state $|1100\rangle+|0011\rangle$. It is plausible that this state can be obtained with at most probability $1 / 2$ using LOP followed by PC. The density matrix for the first two modes is $\rho=|00\rangle\langle 00|+| 11\rangle\langle 11|$. If we consider a state $\mu$ obtained from single photons with linear optics, is it true that the maximum $p$ for which $\mu=p \rho+(1-p) \varrho$ with $\varrho$ a density matrix is $p=1 / 2$ ?

Because of their application to scalable linear optics quantum computation, the postselected gates NS and CS and their variations are being studied both experimentally and theoretically by many researchers. Experimental work preparing for the implementation of these gates has been reported in [6, 7]. Related gates and schemes have been investigated in [8, 9, 10]. Postselection techniques for implementing operations such as the above have been studied in [11, 12, 13]. Related bounds, originally motivated by the problem of realizing a complete Bell-basis measurement can be found in [14, 15, 16].

\section{Acknowledgments}

Many thanks to Leonid Gurvits for stimulating discussions. This work was supported by the DOE (contract W-7405-ENG-36) and the NSA.

[1] E. Knill, R. Laflamme, and G. Milburn, Nature 409, 46 (2001), URL http://www.nature.com/nature/journal/v409/n6816/full/409046a0_fs.html.

[2] D. Gottesman, A. Kitaev, and J. Preskill, Phys. Rev. A 64, 012310/1 (2001).

[3] N. Yoran and B. Reznik (2003), http://www.arxiv.org/abs/quant-ph/0303088

[4] E. Knill, Phys. Rev. A 66, 052306/1 (2002).

[5] M. Reck, A. Zeilinger, H. J. Bernstein, and P. Bertani, Phys. Rev. Lett. 73, 58 (1994).

[6] T. B. Pittman, B. C. Jacobs, and J. D. Franson (2001), quant-ph/0109128, URL http://www.arxiv.org/abs/quant-ph/0109128

[7] T. B. Pittman, B. C. Jacobs, and J. D. Franson, Phys. Rev. A 66, 052305/1 (2002).

[8] T. Rudolph and J.-W. Pan (2001), quant-ph/0108056, URL http://www.arxiv.org/abs/quant-ph/0108056.

[9] T. C. Ralph, A. G. White, W. J. Munro, and G. J. Milburn, Phys. Rev. A 65, 012314/1 (2002), quantph/0108049, URL/http: //www.arxiv.org/abs/quant-ph/0108049.

[10] J. D. Franson, M. M. Donegan, M. J. Fitch, B. C. Jacobs, and T. B. Pittman (2002), quant-ph/0202160, URL http://www.arxiv.org/abs/quant-ph/0202160.

[11] J. Clausen, L. Knöll, and D.-G. Welsch (2003), quant-ph/0303004, URL http://www.arxiv.org/abs/quant-ph/0303004

[12] G. G. Lapaire, P. Kok, J. P. Dowling, and J. E. Sipe (2003), quant-ph/0305152, URL http://www.arxiv.org/abs/quant-ph/0305152

[13] S. Scheel, K. Nemoto, W. J. Munro, and P. L. Knight (2003), quant-ph/0305082, URL http://www.arxiv.org/abs/quant-ph/0305082 
[14] P. van Loock and N. Lütkenhaus (2003), quant-ph/0304057, URL http://www.arxiv.org/abs/quant-ph/0304057

[15] N. Lütkenhaus, J. Calsamiglia, and K.-A. Suominen, Phys. Rev. A 59, 3295 (1999).

[16] J. Calsamiglia (2001), quant-ph/0108108, URL http://www.arxiv.org/abs/quant-ph/0108108 